\title{
FINGERPRINT IMAGE ENHANCEMENT BY DEVELOP MEHTRE TECHNIQUE
}

\author{
Mustafa Salah Khalefa ${ }^{1}$, Zaid Amin Abduljabar ${ }^{2}$ and Huda Ameer Zeki ${ }^{3}$ \\ ${ }^{1}$ Department of computer Science, Education College, Basra University, IRAQ. \\ mustafasalah@yahoo.com \\ ${ }^{2}$ Department of computer Science, Education College, Basra University, IRAQ. \\ zaidalsulami @yahoo.com \\ ${ }^{3}$ Department of computer Science, Shatt Al-arab University College, IRAQ. \\ hzahron@yahoo.com
}

\begin{abstract}
Fingerprint identification is one of the most reliable biometrics technologies. There are many applications of fingerprint recognition such as voting, ecommerce, bank, virtual banks and military. Fingerprint image enhancement is an essential preprocessing step in extract minutiae from the input fingerprint images. In this paper, we propose an image enhancement method by developing Mehter method for directional image. The enhancement is done by added the Block Filtering, Histogram Equalization and High-Pass Filtering. We have evaluated the performance of the enhancement image method by tested it with 100 fingerprint images. Experimental results show the enhancement method improves the recognition more accuracy.
\end{abstract}

\section{KEYWORDS}

Fingerprint, directional image, Histogram equalization, High-pass filter, and, Block filtering.

\section{INTRODUCTION}

Fingerprint recognition is being widely applied in the personal identification for the purpose of high degree of security. However, some fingerprint images captured in variant applications are poor in quality, which corrupts the accuracy of fingerprint recognition. Consequently, fingerprint image enhancement is usually the first step in fingerprint recognition [1]. There have existed a variety of research activities in the field on image enhancement. Mehtre and Murthy [2] and O'Gorman and Nickerson [3] achieved fingerprint image enhancement based on directional image filter. Maio and Maltoni presented direct gray-scale minutiae detection which had low computational complexity [4]. Jiang resorted to an anisotropic filter and an oriented low pass filter to suppress noises [5]. Greenberg, Mayer and Kogan using local histogram equalization, Wiener filtering, and image binarization for Fingerprint Image Enhancement [6]. Tony Lindeberg using scale-space representation with a module for automatic scale selection based on the maximization of normalized derivatives over scales, early visual modules can be made scale invariant [7]. Sepasian, Balachandran and Mares presented a threestep procedure for the fingerprint identification and enhancement, using CLAHE with Clip Limit [8]. Li Wang and Nandita Bhattacharjee proposed propose an adaptive image preprocessing approach that can significantly improve poor quality images according to their noise level based on contrast stretching, power-law transformation and Gabor filter [9]. Poramate 
Prasarn, Keokanlaya Sihalath and Somsak Choomchuay proposed a technique a dynamic enhancement method for an adaptive fingerprint matching [10]. Fingerprint identification accuracy for the both methods which are the conventional method and directional image from gray scale based on fingerprint image quality. Fingerprint image low qualities are caused by $[11,12]$ :

1. Dry or wet skin conditions, improper pressure applied to the sensors, and weak ridge patterns may contain meaningful fingerprint patterns.

2. Scar effect that disrupt ridges and furrow flow.

3. Fingerprint image that contains random noise during image acquisitions.

In fingerprint image enhancement, the directional image usage is very important. Direction for each ridge is obtained by using directional image calculation. The accuracy of ridge direction ensures the successful of fingerprint image enhancement. The production of Directional Image is based on the concept introduced by Mehtre and Murthy (1987) [2]. But, what are the filtering techniques that can be added to enhance a fingerprint image by using directional image? In other words can us rebuilding image without the effects of scars, sweat holes, cuts, bruises, cracks, and calluses and increase the uniformity of the ridges and furrows. These are the objectives of this research. This research will focus on the use of gray scale fingerprint images. The database consists of 60 fingerprint images in various noises.

\section{THE PROPOSED METHOD}

\subsection{Fingerprint Enhancement}

A fingerprint image enhancement method receives an input fingerprint image, applies a set of intermediate steps on the input image, and finally outputs the enhanced image. There are two steps. First one is directional image and the second one is directional Fourier filtering. In this research the process of directional Fourier filtering is still without change. Only the Mehtre technique For Directional Image processing is changed and enhanced. The enhancement is done by added the Block Filtering and, Histogram Equalization and High-Pass Filtering as shown in Figure 1. It reforms the wrong directional element in producing directional image as will discuss in the next section. 


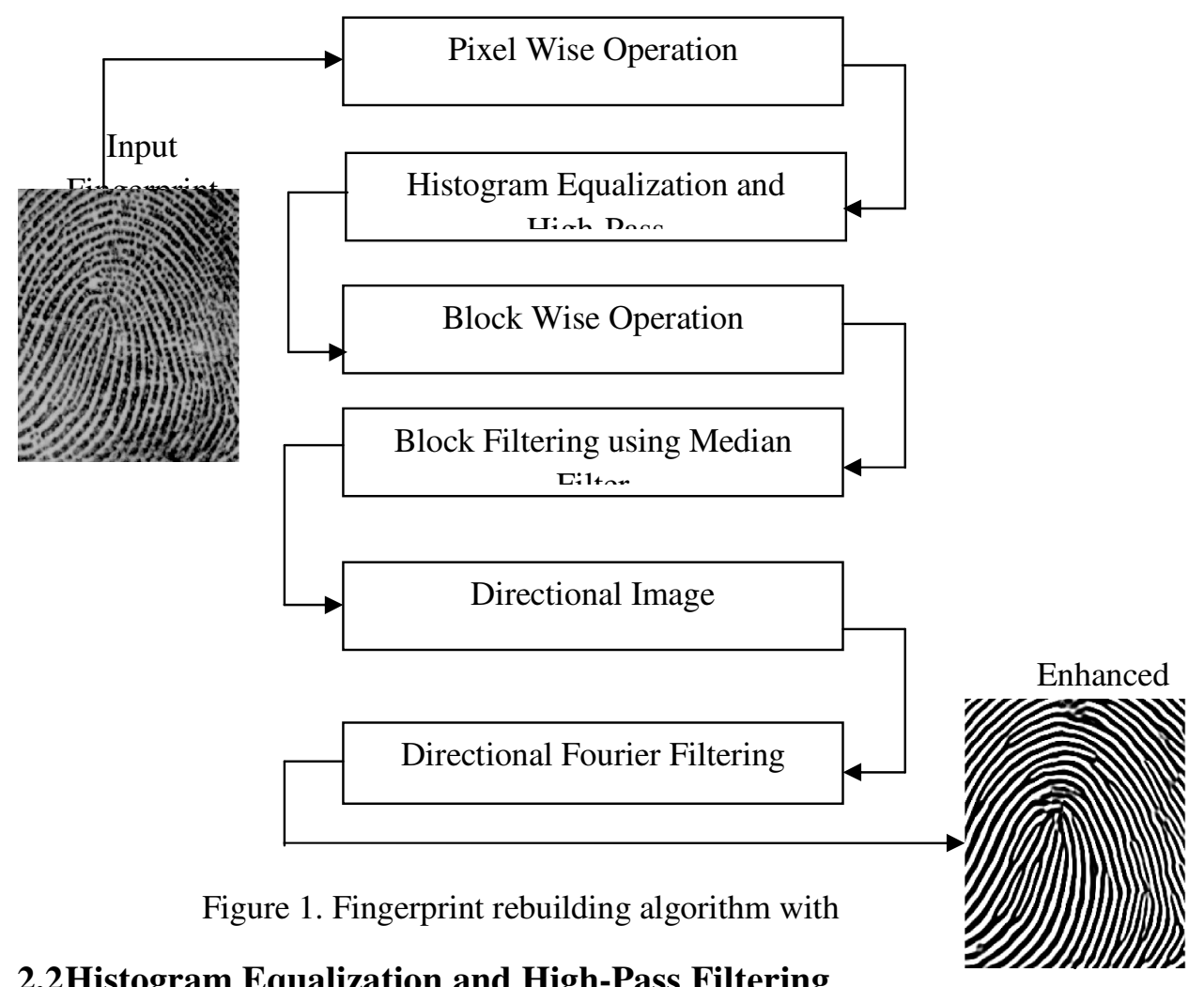

\subsection{Histogram Equalization and High-Pass Filtering}

The goal of histogram equalization is to remap the image gray levels so as to obtain a uniform (flat) histogram in the other words to enhance the image quality [13]. The idea is to change the histogram to one which is uniform; that is that every bar on the histogram is of the same height, or in other words that each grey level in the image occurs with the same frequency. In practice this is generally not possible, although as we shall see the result of histogram equalization provides very good results. Suppose our image has $L$ different grey levels $0,1,2$, $3, \ldots, \mathrm{L}-1$, and that grey level $i$ occurs $n_{i}$ times in the image. Suppose also that the total number of pixels in the image is $n$.To transform the grey levels to obtain a better contrasted image, we change grey level as shown in equation (1) and this number is rounded to the nearest integer $[14,15]$.

$$
\left(\frac{n_{0}+n_{1}+\ldots .+n_{i}}{n}\right)(L-1) 1
$$

For example: Suppose a 3-bit grayscale image has the histogram shown in Table 1 associated with a table of the numbers $n_{i}$ of grey values:

Table 1. Histogram value of original image

\begin{tabular}{|l|l|l|l|l|l|l|l|l|}
\hline Grey levels & 0 & 1 & 2 & 3 & 4 & 5 & 6 & 7 \\
\hline$n_{i}$ & 6 & 14 & 0 & 0 & 7 & 4 & 2 & 18 \\
\hline
\end{tabular}


Advanced Computing: An International Journal ( ACIJ ), Vol.2, No.6, November 2011

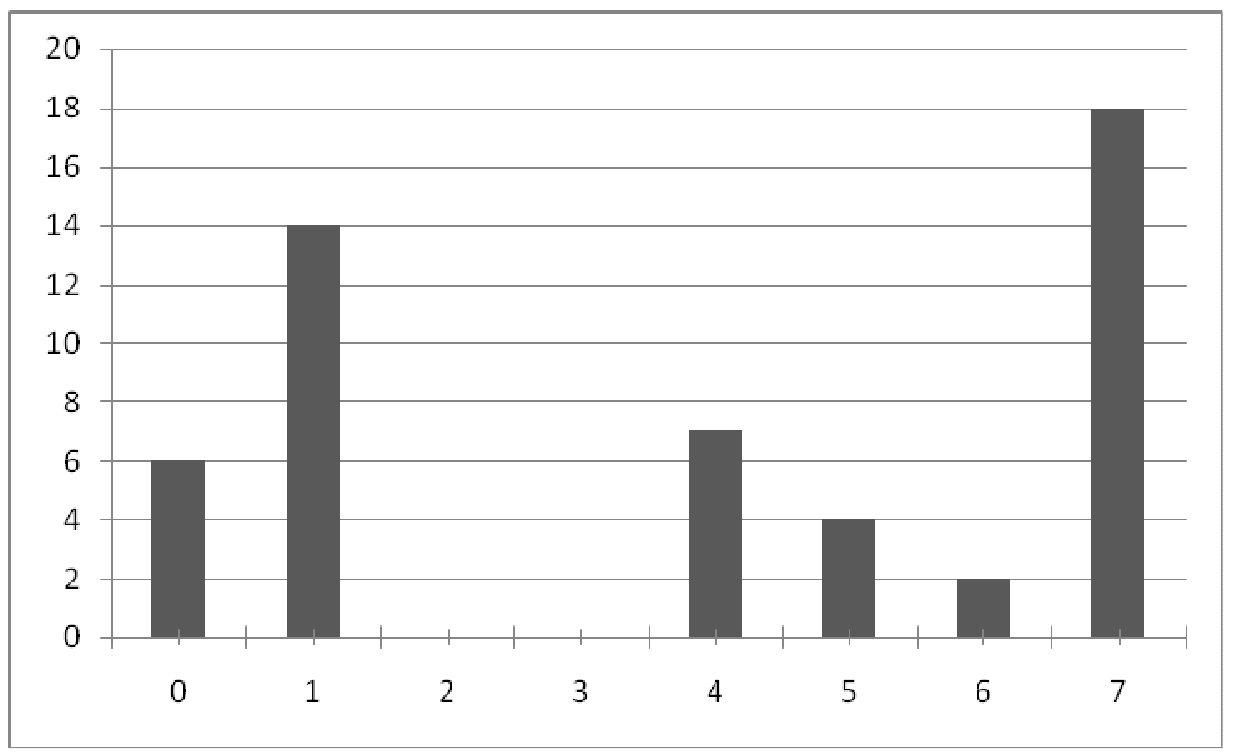

Figure 2. Histogram indicating poor contrast

We would expect this image to be uniformly bright, with a few dark dots on it. To equalize this histogram, we form running totals of the $n_{i}$ and multiply each by (7/51):

Table 2. The histogram value after equalization

\begin{tabular}{|l|l|l|c|l|}
\hline Grey Level $\mathbf{i}$ & $\mathbf{n}_{\mathbf{i}}$ & $\sum \mathbf{n}_{\mathbf{i}}$ & $\mathbf{( 7 / 5 1 )} \sum \mathbf{n}_{\mathbf{i}}$ & Rounded Value \\
\hline 0 & 6 & 6 & 0.823 & 1 \\
\hline 1 & 14 & 20 & 2.745 & 3 \\
\hline 2 & 0 & 20 & 2.745 & 3 \\
\hline 3 & 0 & 20 & 2.745 & 3 \\
\hline 4 & 7 & 27 & 3.705 & 4 \\
\hline 5 & 4 & 31 & 4.254 & 4 \\
\hline 6 & 2 & 33 & 4.529 & 5 \\
\hline 7 & 18 & 51 & 7 & 7 \\
\hline
\end{tabular}




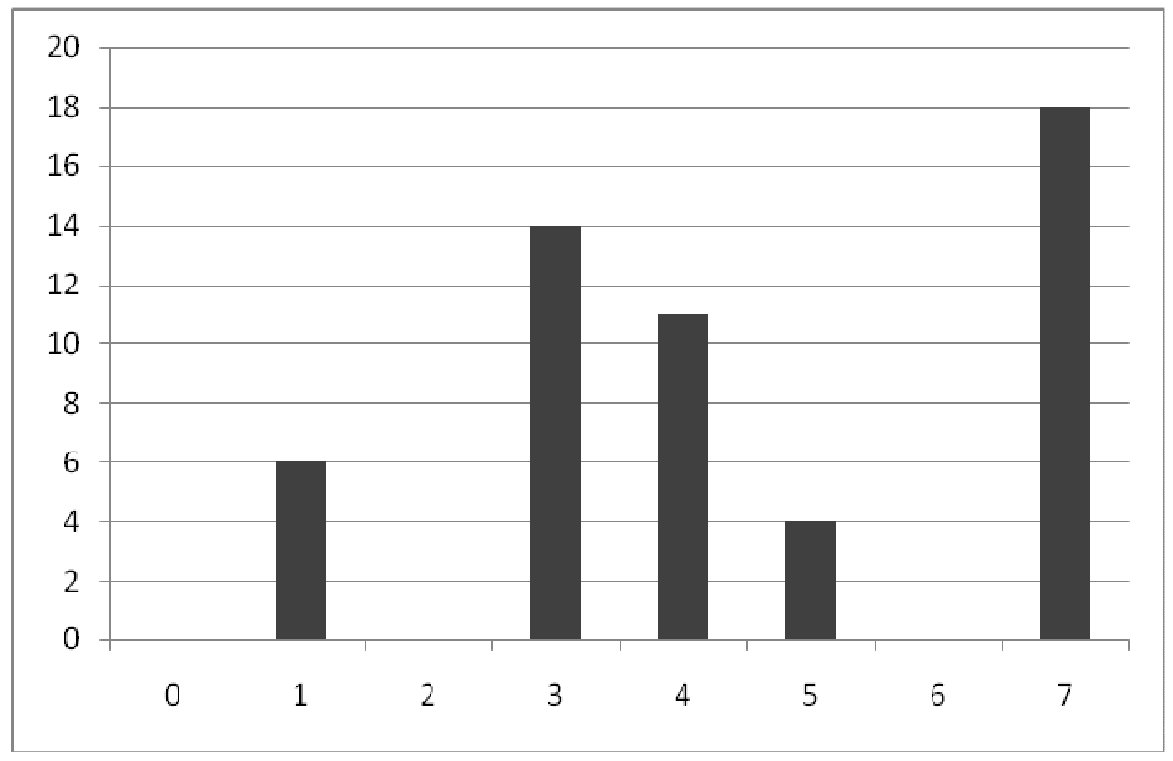

Figure 3. The histogram figure after equalization

We now have the following transformation of grey values, obtained by reading of the first and last columns from the Table 2:

\begin{tabular}{|l|l|l|l|l|l|l|l|l|}
\hline Original Grey levels & 0 & 1 & 2 & 3 & 4 & 5 & 6 & 7 \\
\hline New Grey Level & 1 & 3 & 3 & 3 & 4 & 4 & 5 & 7 \\
\hline
\end{tabular}

Table3. The Original and new Grey Level

And the histogram of the new values is shown in Figure 3. This is far more spread out than the original histogram, and so the resulting image should exhibit greater contrast as shown in Figure 4.

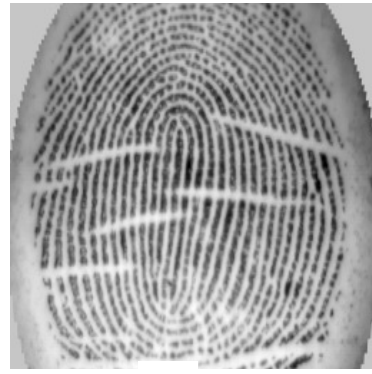

a

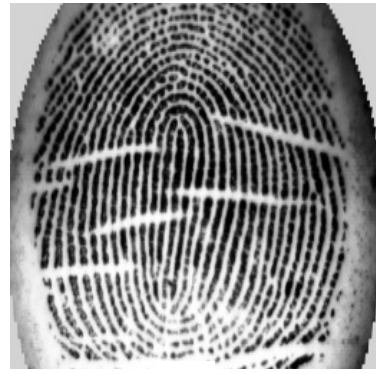

b

Figure4. a) The Original fingerprint b) after Histogram Equalization

High-Pass filtering is accomplished by using a kernel containing a mixture of positive and negative coefficients. An Omni-directional High-Pass filter is that of responses is the same. All the direction, which gray level, varies should have positive coefficients near to its centre 
and negative coefficients in the periphery of the kernel $[14,15]$. Figure 5 shows the HighPass filter used in this research.

\begin{tabular}{|c|c|c|}
\hline 0 & -1 & 0 \\
\hline-1 & 5 & -1 \\
\hline 0 & -1 & 0 \\
\hline
\end{tabular}

Figure 5. A High- Pass spatial mask filter

The movement of High-Pass Filter is overlapping on other pixels. Figure 6 shows the movement of this filter in gray scale fingerprint image.

\begin{tabular}{|c|c|c|cc}
\hline 155 & 123 & 117 & 152 & 189 \\
\cline { 1 - 3 } 161 & 141 & 132 & 145 & 45 \\
\cline { 1 - 3 } 139 & 121 & 134 & 122 & 112 \\
\cline { 1 - 3 } 125 & 158 & 175 & 201 & 168 \\
250 & 145 & 158 & 135 & 177 \\
\cline { 1 - 2 } & & & &
\end{tabular}

\begin{tabular}{l|l|l|l|c}
\cline { 3 - 4 } 1 & \multicolumn{3}{c}{} \\
\cline { 3 - 4 } 155 & 123 & 117 & 152 & 189 \\
161 & 141 & 132 & 145 & 45 \\
139 & 121 & 134 & 122 & 112 \\
125 & 158 & 175 & 201 & 168 \\
\cline { 2 - 4 } 250 & 145 & 158 & 135 & 177
\end{tabular}

\begin{tabular}{|c|c|c|r|r|}
\multicolumn{1}{r|}{155} & 123 & 117 & 152 & 189 \\
\hline 161 & 141 & 132 & 145 & 45 \\
\cline { 1 - 3 } 139 & 121 & 134 & 122 & 112 \\
\hline 125 & 158 & 175 & 201 & 168 \\
\cline { 1 - 3 } 250 & 145 & 158 & 135 & 177
\end{tabular}

\begin{tabular}{l|l|l|l|c|}
155 & 123 & 117 & 152 & 189 \\
\cline { 3 - 4 } 161 & 141 & 132 & 145 & 45 \\
139 & 121 & 134 & 122 & 112 \\
125 & 158 & 175 & 201 & 168 \\
\cline { 3 - 4 } 250 & 145 & 158 & 135 & 177
\end{tabular}

Figure 6. High-Pass Filter movements from left to right and from top to down in fingerprint image

Figure 6 above, the first $3 \times 3$ window contains 155, 123, 117, 161, 141, 132, 139, 121 and 134 using the mask in Figure 5. Value 141 is the center of the windows and this value will be replaced with a new value. The calculation of High-Pass Filter is : $\mathrm{t}=155 * 0+(-1 * 123)+117 * 0+(-1 * 161)+(5 * 141)+(-1 * 132)+139 * 0+(-1 * 121)+134 * 0=$ 168

To get positive gray level value between 0 and 255, the conditions must be followed:

1. If $\mathrm{t}>255, \mathrm{t}=255$

2. If $\mathrm{t}<0, \mathrm{t}=0$

3. Else use original t value 
By referring to the condition, the example meets the condition number three. As a result the new value in $3 \times 3$ window is shown in Figure 7 Below:

\begin{tabular}{|l|l|l|}
\hline 155 & 123 & 117 \\
\hline 161 & 168 & 132 \\
\hline 139 & 121 & 134 \\
\hline
\end{tabular}

Figure 7. The gray scale value after High-Pass Filtering

Histogram Equalization and High-Pass Filter is used in this research because this methods give a good result in fixing wrong directional element in directional image.

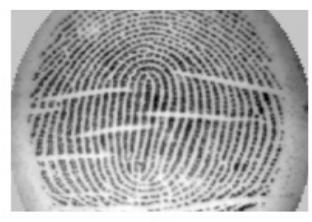

(a)

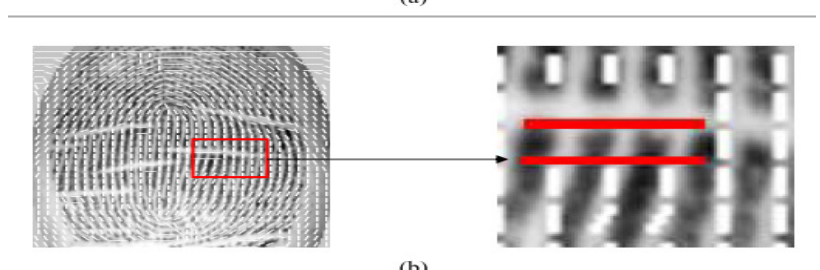

(b)

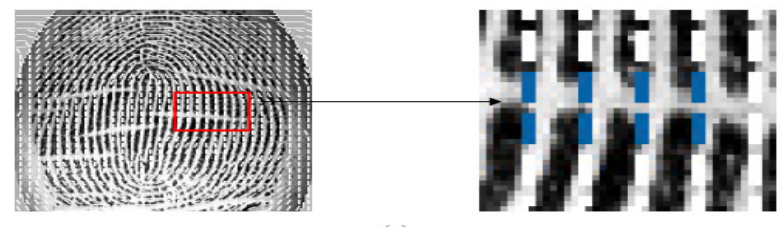

(c)

From the result in Figure 8, it shows that the scars affect in fingerprint image disrupts

Figure 8. a) Original fingerprint image, b) Without filtering c) Using Histogram

directional element in Directional Image. Thus, with wrong Directional Image, the percentage of false minutiae creation and detection is increased. The usage of Histogram Equalization and High-Pass in increasing the fingerprint minutiae and contrast is very useful in obtaining accurate directional elements.

\subsection{Block Filtering Using Median Filter}

The presence of noise after Block Wise stage causes, the Block Directional Image to be not correct most of the time. Figure 9 shows the Block Directional Image after Block Wise Operation containing false block direction. 

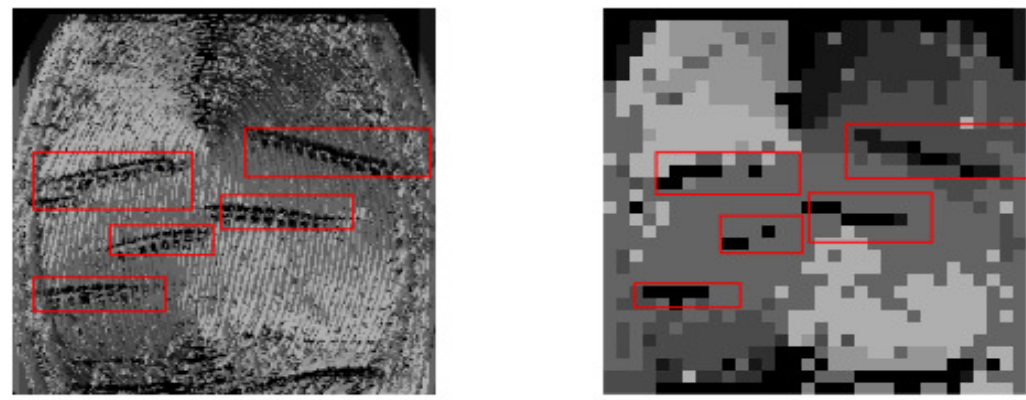

Figure 9 False block direction in block directional image

From the Figure 9, it shows the wrong block direction caused by the effect of scars in fingerprint image. To overcome this problem, a Median Filter is used to filter out the wrong element in Block Directional Image.

Median Filter may be used if the objective is to achieve noise reduction with a minimum amount of blurring $[14,15,16]$. That is, the gray level of each pixel is replaced by the median of the gray levels in the neighborhood of that pixel. This method is particularly effective when the noise pattern consists of strong, spike like components and, the characteristic that is to be preserved is the edge sharpness $[14,15]$.

In order to perform median filtering in a neighborhood of a pixel, sort the values of the pixel and its neighbors, determine the median, and assign this value to the pixel. For example in a $3 \times 3$ neighborhood, the median is the 5 th largest value, in a $5 \times 5$ neighborhood the median is the 13th largest value and so on. In this research, we used $3 \times 3$ windows or neighborhood for Median Filtering. For example, in Figure 14.a, the 3x3 neighborhood has a value of $(64,64,64,64,255,255,64,64$, and 255). These value are sorted as $(64,64$, $64,64,64,64,255,255,255)$, which results in a median of 64 . Figure 10 shows the pixels value and new fingerprint image after median filtering.

\begin{tabular}{|c|c|c|}
\hline 64 & 64 & 64 \\
\hline 64 & 255 & 255 \\
\hline 64 & 64 & 255 \\
\hline
\end{tabular}

a

\begin{tabular}{|c|c|c|}
\hline 4 & 64 & 64 \\
\hline 64 & 64 & 255 \\
\hline 64 & 64 & 255 \\
\hline
\end{tabular}

b

Figure 10. a) Original $3 \times 3$ neighborhood, b) after Median Filter 


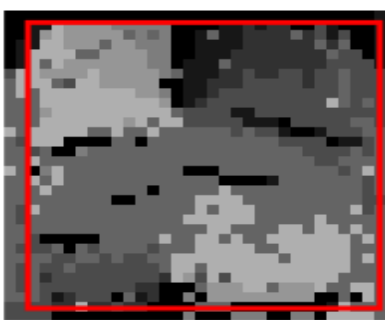

(a)

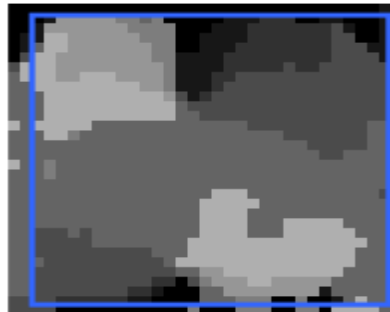

(b)

Figure 11. Directional image a) before Median filter b) after Median filter

Figure 11.a displays block directional image with full of noise inside the red rectangle. This noise will produce a wrong directional element in directional image and it leads to the false minutiae extraction. Figure 11.b shows the effect Median Filter. In Figure 11.b, the block directional image is cleaned and it is free from noise. The effect of Median Filtering is shown in Figure 12 below.

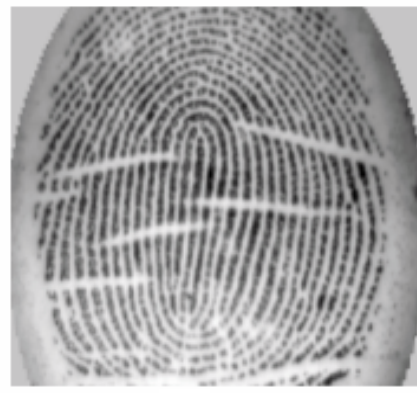

a

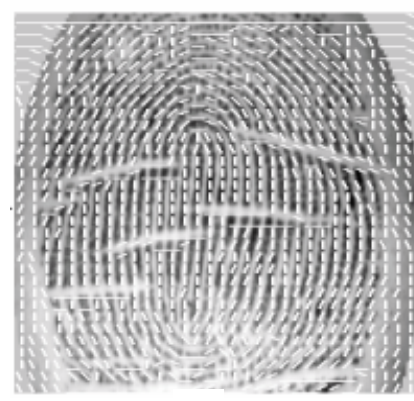

$\mathrm{b}$

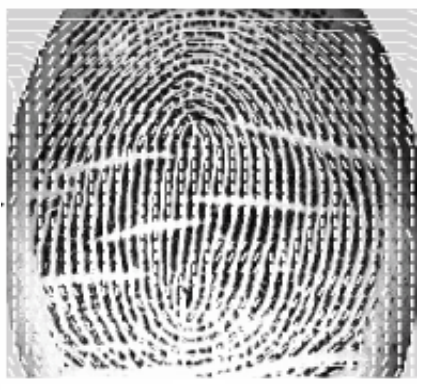

c

Figure 12. a) Original fingerprint image b) Before Median filter c) After Median filter

elements and by looking at the original 1mage, these wronged elements are caused by scar effect. The wrong elements will increase in the percentage of having false minutiae extraction. Then are fixed through Median filter as shown in Figure 12.c. It displays a better Directional Image. Using Median Filter all the wrong elements are replaced with the correct ones. Figure 13 below shows eight set pre-rebuilding fingerprint image using Directional Fourier Filtering in eight directions obtain from Directional Image.

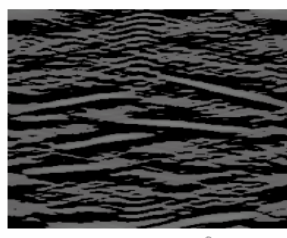

Direction $0^{\circ}$

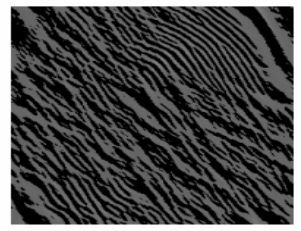

Direction $45^{\circ}$

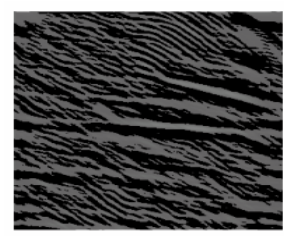

Direction $22.5^{0}$

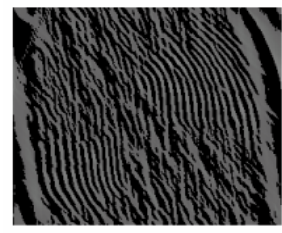

Direction $67.5^{\circ}$

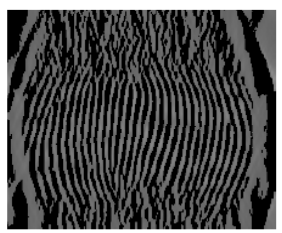

Direction $90^{\circ}$

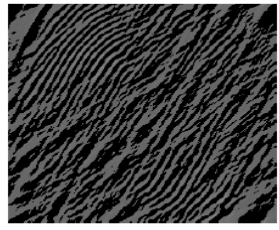

Direction $135^{\circ}$

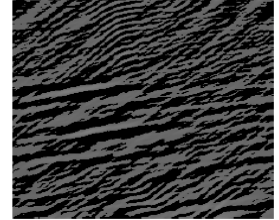

Direction $112.5^{\circ}$

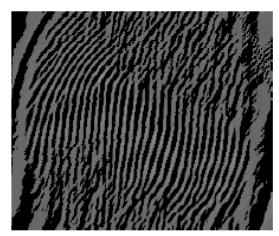

Direction $157.5^{\circ}$

Figure 13. Eight set pre-rebuilding fingerprint image 


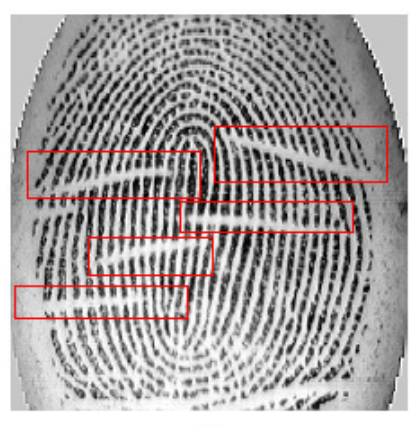

(a)

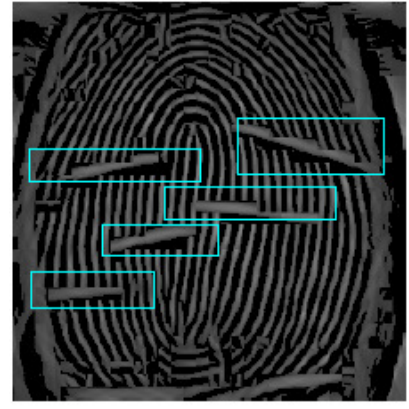

(b)

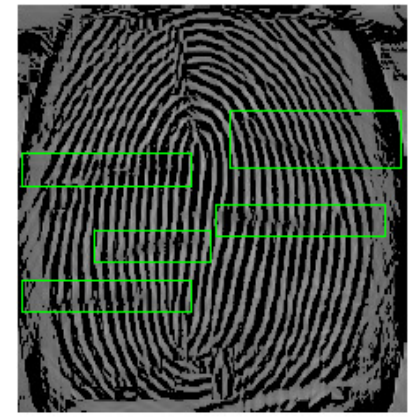

(c)

Figure 14. Rebuilding of fingerprint image a) Original fingerprint b) Original directional image

c) Enhanced directional image

\section{EXPERIMENTAL RESULT AND DISCUSSION}

The main aim of this experiment is to compare the fingerprint rebuilding using existing directional image with enhanced directional image. A test sample selection is important before the experiment is held. The suitable samples determine the result. Thus, fingerprint image quality plays an important role in fingerprint rebuilding. To verify the effectiveness of Proposed Method, a comparative experiment is performed. There are two methods for this experiment: the first one is fingerprint rebuilding based on directional image by using existing Mehtre Technique. And the second one is fingerprint rebuilding based on directional image by using enhanced Mehtre Technique.

Table4. Result of fingerprint rebuilding for all types

\begin{tabular}{|c|c|c|c|c|}
\hline Method & $\begin{array}{c}\text { Arch } \\
\text { Types }\end{array}$ & $\begin{array}{c}\text { Left Loop } \\
\text { Types }\end{array}$ & $\begin{array}{c}\text { Right Loop } \\
\text { Types }\end{array}$ & Tended types \\
\hline Mehtre Technique & $65 \%$ & $68.4 \%$ & $67 \%$ & $58 \%$ \\
\hline Improved Mehtre Technique & $85.5 \%$ & $82 \%$ & $81 \%$ & $77 \%$ \\
\hline
\end{tabular}

\subsection{Conclusion}

Based on the result of the experiment phase in this research we found. Firstly, the use of Histogram Equalization and High-Pass Filter enable to increase fingerprint contrasts and enhance ridges and furrows structures to get accurate directional element for each ridges. Secondly, by using Median Filter in Block Filtering, it enables to filter and correct wrong block direction and removes noise getting from Block Wise Operation.

As conclusion, the enhanced Mehtre Technique produces a fine fingerprint image quality comparing with existing Mehtre Technique. 
Advanced Computing: An International Journal ( ACIJ ), Vol.2, No.6, November 2011

\section{REFERENCES}

[1] Jianwei Yang, Lifeng Liuand Tianzi Jiang and Yong Fan, , (2003), “A modified Gabor filter design method for fingerprint image enhancement", Elsevier Journal, Pattern Recognition Letters 24, pp. 1805-1817.

[2] B.M. Mehtrea, N.N. Murthya, S. Kapoora and B. Chatterjeeb, (1987), "Segmentation of fingerprint images using the directional image", Elsevier Journal, Pattern Recognition Vol. 20, No. 4, pp. 429-435.

[3] O'Gorman L., and Nickerson, J.V., (1989), “An Approach to Fingerprint Filter Design, Pattern Recognition”, Vol. 22, No. 1, pp. 29-38.

[4] Dario Maio and Davide Maltoni, (1997), "Direct Gray-Scale Minutiae Detection in Fingerprints", IEEE transactions on pattern analysis and machine intelligence, Vol. 19, No. 1, pp. $27-40$.

[5] Jiang X., (2001), “A study of fingerprint image filtering”. In: Proc. 8th Internet Conf. on Image Processing (Biometrics), Thessaloniki, Greece, pp. 238-241

[6] Shlomo Greenberg, Mayer Aladjem and Daniel Kogan, (2002), "Fingerprint Image Enhancement using Filtering Techniques”, Elsevier Science Ltd, Real-Time Imaging 8, pp. 227-236.

[7] Tony Lindeberg, (2009), "Scale-space”, In: Encyclopedia of Computer Science and Engineering (Benjamin Wah, ed), John Wiley and Sons, Vol. 5, pp. 2495-2504.

[8] M. Sepasian, W. Balachandran and C. Mares, (2008), "Image Enhancement for Fingerprint Minutiae-Based Algorithms Using CLAHE, Standard Deviation Analysis and Sliding Neighborhood", Proceedings of the World Congress on Engineering and Computer Science 2008 WCECS 2008, October 22 - 24, USA. pp. 479-491.

[9] Li Wang and Nandita Bhattacharjee, (2010), "Adaptive approach to fingerprint image enhancement", Proceedings of the 8th International Conference on Advances in Mobile Computing and Multimedia, ACM New York, NY, USA. pp. 323- 336.

[10] Poramate Prasarn, Keokanlaya Sihalath and Somsak Choomchuay, (2010), "A DYNAMIC ENHANCEMENT METHOD FOR FINGERPRINT MATCHING”, ", Proceedings of the 3rd Biomedical Engineering International Conference, Kuala Lumpur, Malaysia, pp. 237 - 241.

[11] Henry C. Lee and R. E. Gaensslen, (2001), “Advances in Fingerprint Technology”, CRC Press, USA.

[12] Peter. Komarinski, (2005) “AUTOMATED FINGERPRINT IDENTIFICATION SYSTEMS (AFIS”), Elsevier Academic Press, USA.

[13] Jagdish Lal Raheja and Umesh Kumar, (2010), "Human facial expression detection from detection in captured image using back propagation neural network", International Journal of computer science and Information Technology (IJCSIT), Vol. 2, No. 1, pp. 116- 123. 
Advanced Computing: An International Journal ( ACIJ ), Vol.2, No.6, November 2011

[14] Qiang Wu, Fatima A. Merchant and Kenneth R. Castleman, (2008), "Microscope Image Processing”, Elsevier Inc, U.K.

[15] A. Bovik, (2009), "The Essential Guide to image processing”, Elsevier Inc., USA.

[16] P.Ramakrishna Rao, Dr.B.Addai, G.Ramakrishna and T.PanduRanga Vital, (2011), “Adaptive Fuzzy Filtering for Artifact Reduction in Compressed images and videos", International Journal of Computer Science \& Information Technology (IJCSIT), Vol. 3, No. 1, pp. 118- 137. 